\title{
Intraocular involvement is associated with a high risk of disease relapse in primary central nervous system lymphoma
}

\author{
LIN ZHUANG $^{1 *}$, JIE LAI $^{2 *}$, KUN CHEN $^{3}$, TIANLING DING ${ }^{1}$, YAN YUAN $^{1}$, YAN MA $^{4}$, \\ HUI KANG ${ }^{4}$, ZHIGUANG LIN ${ }^{1,4}$, NI FAN ${ }^{1}$, JINGJING MA ${ }^{4}$, QINGCHAO ZENG ${ }^{4}$, \\ XIAOPING XU ${ }^{1}$, QINGPING WANG ${ }^{2}$ and BOBIN CHEN ${ }^{1,4}$ \\ Departments of ${ }^{1}$ Hematology and ${ }^{2}$ Ophthalmology, Huashan Hospital; \\ Departments of ${ }^{3}$ Laboratory Medicine and ${ }^{4}$ Hematology, Huashan Hospital North, \\ Fudan University, Shanghai 200040, P.R. China
}

Received March 27, 2018; Accepted October 1, 2018

DOI: $10.3892 /$ or.2018.6781

\begin{abstract}
The aim of the present study was to prospectively evaluate the recurrence and survival outcome of primary central nervous system lymphoma (PCNSL) with intraocular involvement. For this purpose, a prospective cohort of 103 pathologically confirmed patients with PCNSL was enrolled in this study. Ophthalmologic examinations were performed both at diagnosis and during follow-up. The patients with PCNSL with suspected intraocular involvement underwent vitrectomy for confirmation. Patients who presented with intraocular involvement either at diagnosis or during disease progression were allocated to the intraocular lymphoma (IOL) group. All patients with PCNSL received systemic methotrexate (MTX)-based chemotherapy with or without radiotherapy. MTX intravitreal injection combined with systemic MTX-based chemotherapy was recommended once ocular lesions were confirmed. Recurrent intraocular and central nervous system (CNS) events, progression-free survival (PFS) and overall survival (OS) outcomes were analyzed. The findings of this study revealed that 21 patients with PCNSL exhibited intraocular involvement. One patient with IOL presented with isolated ocular lymphoma at the initial diagnosis, and the others presented with ocular involvement along
\end{abstract}

Correspondence to: Dr Bobin Chen, Department of Hematology, Huashan Hospital, Fudan University, 12 Urumuqi Road (middle), Shanghai 200040, P.R. China

E-mail: bbchen@fudan.edu.cn

Dr Qingping Wang, Department of Ophthalmology, Huashan Hospital, Fudan University, 12 Urumuqi Road (middle), Shanghai 200040, P.R. China

E-mail: wangqingping71@163.com

*Contributed equally

Key words: intraocular lymphoma, primary central nervous system lymphoma, relapse, progression-free survival, overall survival with CNS invasion during the course of the disease. A total of 14 patients received systemic MTX-based chemotherapy prior to the diagnosis of IOL. The recurrence rates in patients with or without intraocular involvement were 71.4 and $46.3 \%$, respectively $(\mathrm{P}=0.04)$ and the relapse sites in the patients with IOL included the brain ( 3 patients), eyes ( 6 patients), and both brain and eyes (6 cases). The median PFS was 13 months in the IOL group and 19 months in the patients without intraocular involvement (non-IOL) $(\mathrm{P}=0.019)$. The median OS was 51 months vs. 56 months, respectively $(\mathrm{P}=0.312)$. There was no significant difference in the 2-year PFS and OS rates between the 2 groups ( $23.8 \%$ vs. $23.2 \%, \mathrm{P}=0.951$; and $61.9 \%$ vs. $41.4 \%$, $\mathrm{P}=0.093$, respectively). On the whole, the findings of this study suggest that patients with IOL have a high risk of relapse and a poor PFS compared to patients without IOL, but a similar OS.

\section{Introduction}

Intraocular lymphoma(IOL) represents a malignant, extranodal form of non-Hodgkin lymphoma (NHL) mainly located in the vitreous, retina and optic nerve head. It is a subtype of primary central nervous system lymphoma (PCNSL) that occurs in isolation [termed primary IOL (PIOL)] or with a simultaneous intracerebral mass (1). IOL can develop either as an isolated manifestation or as a recurrent disease. Approximately $15-25 \%$ of patients with PCNSL present with intraocular involvement at diagnosis or during the follow-up period (2), accounting for $1 \%$ of intracranial tumors. Vitreoretinal lymphoma is the main type of IOL.

IOL is a typical masquerade syndrome and shares some symptoms, such as floaters and blurred vision, with posterior uveitis, vitritis and retinitis (3). These shared symptoms can result in difficult or delayed diagnoses. Approximately $80 \%$ of patients with IOL have bilateral eye involvement (4). Although examinations, including optical coherence tomography (OCT) and B-type ultrasonography can provide valuable insight for diagnosis, cytological analysis is an indispensable method for diagnostic confirmation. The main pathological type is diffuse large B cell lymphoma. 
It has to be determined whether IOL should be considered a distinct lymphoma entity. For the rarity of the disease, little is known about the differences between IOL and PCNSL without intraocular involvement (non-IOL). An increasing number of studies have suggested that IOL and PCNSL are distinct in terms of genetic features and recommended clinical actions $(5,6)$. Additionally, Wang et al (7) used a single nucleotide polymorphism microarray to demonstrate that patients with IOL with central nervous system (CNS) lesions at diagnosis had distinct genetic characteristics compared with patients with isolated PIOL and secondary IOL following systemic lymphoma, which may reveal the high heterogeneity and distinctive biological behavior of the disease. Recent studies have concentrated on isolated PIOL $(8,9)$, concluding that it is a less aggressive form of the disease $(10,11)$. However, to the best of our knowledge, only a few studies to date have focused on IOL with CNS involvement or following intracerebral mass due to the difficulty of diagnosis and rarity of the disease $(12,13)$. As a result, questions concerning the biological behavior of IOL remain unresolved.

In this study, we hypothesized that patients with PCNSL with intraocular involvement would have a higher rate of relapse for the following reasons: i) It is difficult to detect intraocular infiltration due to atypical symptoms; ii) the unique genetic makeup of invading tumor cells may result in treatment insensitivity; and iii) intravenous chemotherapy with high-dose methotrexate (HD-MTX) is insufficient for the treatment of IOL due to delivery impediments caused by the blood-ocular barrier. In this study, we focus on the systemic impact of intraocular involvement on the prognosis of patients with PCNSL.

\section{Patients and methods}

Patient eligibility. From October, 2009 to October, 2016, 103 patients with PCNSL were consecutively enrolled at our Huashan Hospital and Huashan Hospital North, Shanghai, China. Their clinical data were prospectively analyzed. All PCNSL diagnoses were confirmed by pathological examinations following stereotactic puncture or surgical tumor reduction. Brain magnetic resonance imaging (MRI), systemic computed tomography (CT) scans, positron emission tomography-CT (PET/CT), bone marrow biopsy and Karnofsky performance scores (KPS) were obtained to aid the diagnosis and evaluation of the disease state. Cerebrospinal fluid (CSF) samples were obtained when it could be done safely and without the possibility of herniation from increased intracranial pressure. The following patients were excluded in the research: i) Patients with systemic lymphoma; ii) patients with severe lesions affecting cardiac, liver or renal function; iii) HIV-positive and immunosuppressed patients; and iv) patients with PCNSL suspected to have intraocular involvement, but who rejected vitreous biopsy.

All patients with PCNSL were screened by ophthalmological assessment (including slit-lamp examination, OCT and ophthalmologic B-ultrasound) when they were first admitted to our hospital. Routine ophthalmical testing was also performed after every 3 chemotherapeutic cycles. Vitreous biopsies were performed when the above-mentioned inspections suggested IOL. The diagnosis of IOL was made when lymphoma cells were found in the vitreous fluid. The identified patients were divided into the IOL and non-IOL groups according to their intraocular involvement status during disease progression.

Blood samples were collected from patients under aseptic precautions and were subjected to centrifugation for $4 \mathrm{~min}$ at $600 \mathrm{x} \mathrm{g}$ after the blood coagulated. The serum was then separated and stored at $4{ }^{\circ} \mathrm{C}$ in refrigerators. The serum lactate dehydrogenase (LDH) concentration was measured using the Lactate Dehydrogenase acc. to IFCC ver.2 (LDHI2) kit (Roche Diagnostics GmbH, Mannheim, Germany). The serum $\beta 2$-microglobulin ( $\beta 2-\mathrm{MG})$ concentration was measured using a latex enhanced immunoturbidimetric assay and tests were directed by the manufacturer of the kit (Zybio, Chongqing, China). All the measurements were performed on an Roche cobas 8000 modular analyzer series at Huashan Hospital. Tumor tissues were fixed in $4 \%$ formalin, embedded in paraffin and sectioned at 4 microns. The slices were deparaffinized in xylol and rehydrated in graded alcohol. Antigen retrieval with $10 \mathrm{mM}$ citrate buffer $(\mathrm{pH} 6.2)$ at $95^{\circ} \mathrm{C}$ was used. After the blocking of non-specific antibody binding by incubating with 5\% BSA, the sections were incubated with anti-Ki-67 monoclonal antibody at a pre-diluted concentration (cat. no. MAB-0672; Maixin Biotechnologies, Fuzhou, China) at room temperature for $45 \mathrm{~min}$ and HRP-conjugated secondary antibody for $1 \mathrm{~h}$ at $37^{\circ} \mathrm{C}$. To reveal endogenous peroxidase activity, the slides were incubated with DAB substrate and stained with hematoxylin. All the Ki-67 stained slides were scanned by microscopy.

Treatment. The patients were treated with HD-MTX-based chemotherapy with or without whole-brain radiotherapy (WBRT). Rituximab, idarubicin (IDA) and teniposide (VM-26) were also administered to the patients with PCNSL in combination with HD-MTX and/or radiotherapy. In total, 16 patients received intravitreal injections of MTX following the identification of intraocular involvement. They were initially treated with intravitreal injections of MTX $(400 \mu \mathrm{g} / 0.1 \mathrm{ml})$ in the affected eyes twice a week for 4 weeks, then once a week for 8 weeks, and finally once a month for 9 months. An intrathecal injection of MTX was administered to patients with meningeal involvement. Patients received salvage drugs (such as high-dose Ara-C and pemetrexed) or radiotherapy at first relapse.

This study received approval from the Ethics Committee of the Institutional Review Board of Huashan Hospital, Fudan University. All the patients voluntarily participated in this study and provided informed consent.

Statistical analysis. Progression-free survival (PFS) was defined as the time from entry into the study to the first sign of disease progression, and overall survival (OS) was defined as the time from study entry to death. The Mantel-Haenszel Pearson Chi-square test or Fisher's exact test were used to evaluate the differences in categorical data between the 2 groups. PFS, OS and 95\% confidence interval (CI) values for PFS and OS were estimated using the Kaplan-Meier method. The log-rank test was used to perform univariate analyses. Variables with a univariate analysis $\mathrm{P}$-value $<0.2$ were examined using the Cox proportional hazards regression model for multivariate analysis. Results were considered statistically 
Table I. Characteristics of patients with PCNSL with and without intraocular involvement.

\begin{tabular}{|c|c|c|c|}
\hline Characteristic & Non-IOL (n=82) & $\operatorname{IOL}(\mathrm{n}=21)$ & P-value \\
\hline Follow-up time, median (range) & $20(3-89)$ & $27(4-83)$ & 0.842 \\
\hline Age, median (range) & $55.5(23-75)$ & $55(37-71)$ & 0.947 \\
\hline$\geq 60$ years & 27 & 4 & 0.216 \\
\hline \multicolumn{4}{|l|}{ Sex } \\
\hline Male & 56 & 12 & \multirow[t]{2}{*}{0.336} \\
\hline Female & 26 & 9 & \\
\hline KPS, median (range) & $70(30-90)$ & $60(30-80)$ & \\
\hline $30-40$ & 28 & 8 & 0.735 \\
\hline$>40$ & 54 & 13 & \\
\hline \multicolumn{4}{|l|}{ Diagnosis } \\
\hline Surgery & 40 & 11 & \multirow[t]{2}{*}{0.768} \\
\hline Biopsy & 42 & 10 & \\
\hline \multicolumn{4}{|l|}{ Lesions } \\
\hline Single & 35 & 13 & \multirow[t]{2}{*}{0.115} \\
\hline Multiple & 47 & 8 & \\
\hline \multicolumn{4}{|l|}{ Initial therapy } \\
\hline Chemotherapy & 47 & 14 & \multirow[t]{2}{*}{0.437} \\
\hline Chemotherapy+radiotherapy & 35 & 7 & \\
\hline \multicolumn{4}{|l|}{$\mathrm{Ki}-67$} \\
\hline \multirow[t]{2}{*}{ Median (range) } & $60(0-95)$ & 75 (20-99) & \\
\hline & $48 / 82$ & $10 / 21$ & 0.350 \\
\hline$\geq 80$ & $16 / 48$ & $5 / 10$ & 0.318 \\
\hline \multicolumn{4}{|l|}{$\mathrm{LDH}$} \\
\hline Median (range) & $203.8(109-638)$ & $182(139-385)$ & 0.763 \\
\hline Elevated $\beta$ 2-MG & $24 / 54$ & $3 / 14$ & 0.117 \\
\hline Meningeal involvement & $16 / 40$ & $5 / 12$ & 0.918 \\
\hline Disease relapse & 38 & 15 & 0.04 \\
\hline Brain & 38 & 3 & \\
\hline Eye & 0 & 6 & \\
\hline Brain and eye & 0 & 6 & \\
\hline Time to diagnosis, median (range) & $1.0(0.2-27.0)$ & $1.0(0.5-16.0)$ & 0.775 \\
\hline
\end{tabular}

PCNSL, primary central nervous system lymphoma; IOL, intraocular lymphoma; KPS, Karnofsky performance scores; LDH, lactate dehydrogenase; $\beta 2-\mathrm{MG}, \beta 2$-microglobulin.

significant at $\mathrm{P} \leq 0.05$. The statistical tests were conducted using STATA 12.0 software.

\section{Results}

Patient characteristics. Of the 103 patients with PCNSL eligible for enrollment into this study, 21 patients $(20.4 \%$, including 12 males and 9 females) presented with intraocular involvement as confirmed by the cytomorphological examination of vitreous aspirate (Fig. 1). The median follow-up period was 21 months (ranging from 3 to 89 months). The mean age at the onset of IOL was 55 years (ranging from 37 to 71 years). The main clinical characteristics of the eligible patients are summarized in Table I. No significant differences were observed between the patients with and without intraocular involvement as regards age, sex, clinical presentations and laboratory data (including Ki-67, serum LDH and $\beta_{2}-\mathrm{MG}$ ).

Characteristics of IOL. Among the 21 patients with IOL, 11 patients had bilateral eye involvement and 10 patients had unilateral eye involvement over the course of the disease. In total, 16 patients had clinical symptoms including the following: Blurred vision, photophobia, fundus hemorrhage, floaters, ocular pain and ocular movement limits. However, 5 patients were asymptomatic when diagnosed. A total of 7 patients presented with IOL at the onset of diagnosis, and 14 patients presented with intraocular involvement along the disease course. In total, 20 patients had a contaminant CNS mass during the course of the disease, and 6 had both parenchymal and ocular involvement at diagnosis. Only 1 patient 

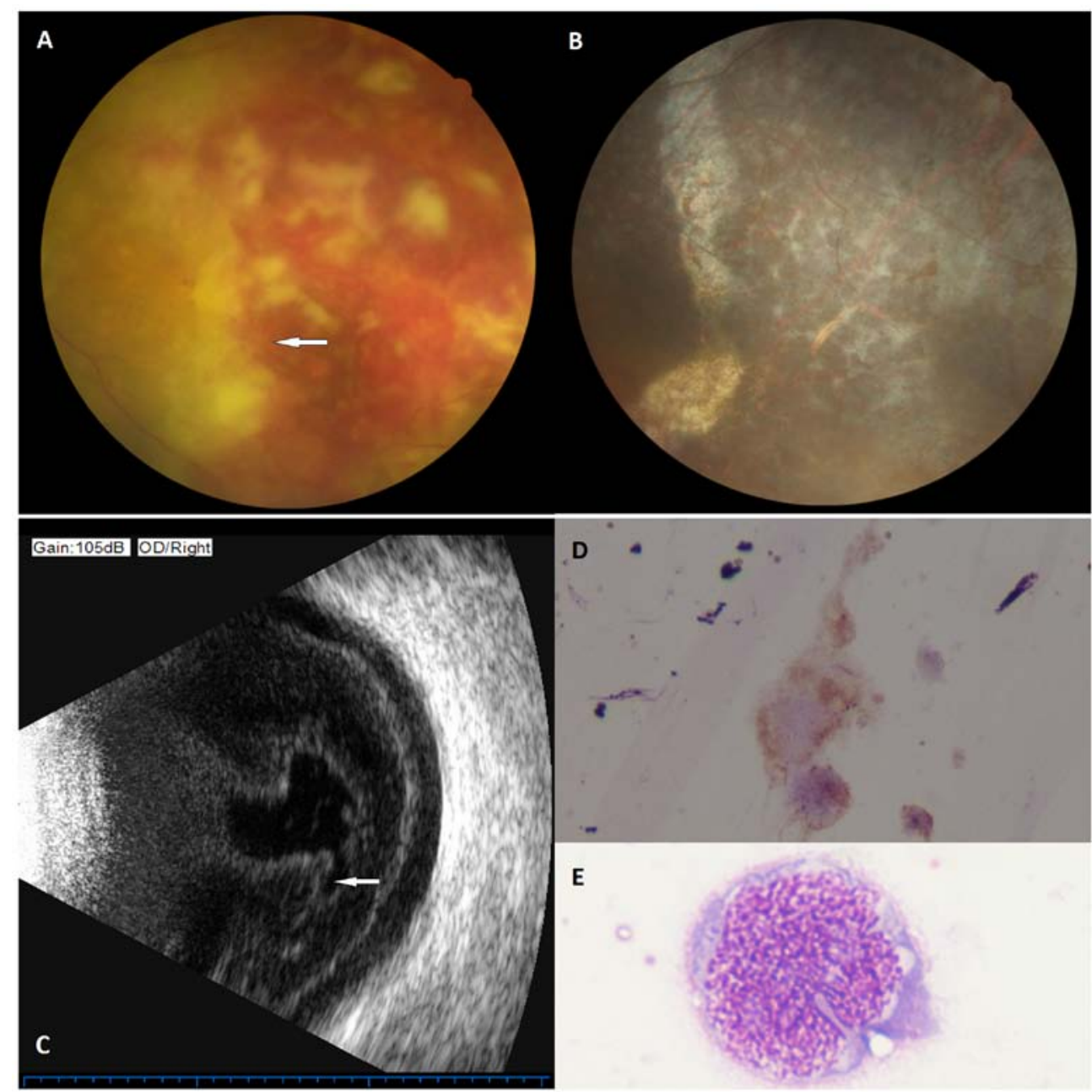

Figure 1. B-scan ultrasound, slit-lamp examination and pathological results of patients with PCNSL with intraocular involvement. (A) Fundus color images demonstrating orange deposits and hemorrhage (white arrow) in the retina before MTX intravitreal injection. (B) Fundus color images showing disappeared orange deposits in the retina after MTX intravitreal injection. (C) Ocular B-scan ultrasound images of a confirmed patient with IOL showing a dense punctate mass in the vitreous cavity. (D) Immunocytology displaying tumor cell expression of CD20 (magnification, x100). (E) Vitrectomy specimen showing lymphocytic blasts with large nuclei and prominent nucleoli (magnification, x400). PCNSL, primary central nervous system lymphoma; IOL, intraocular lymphoma.
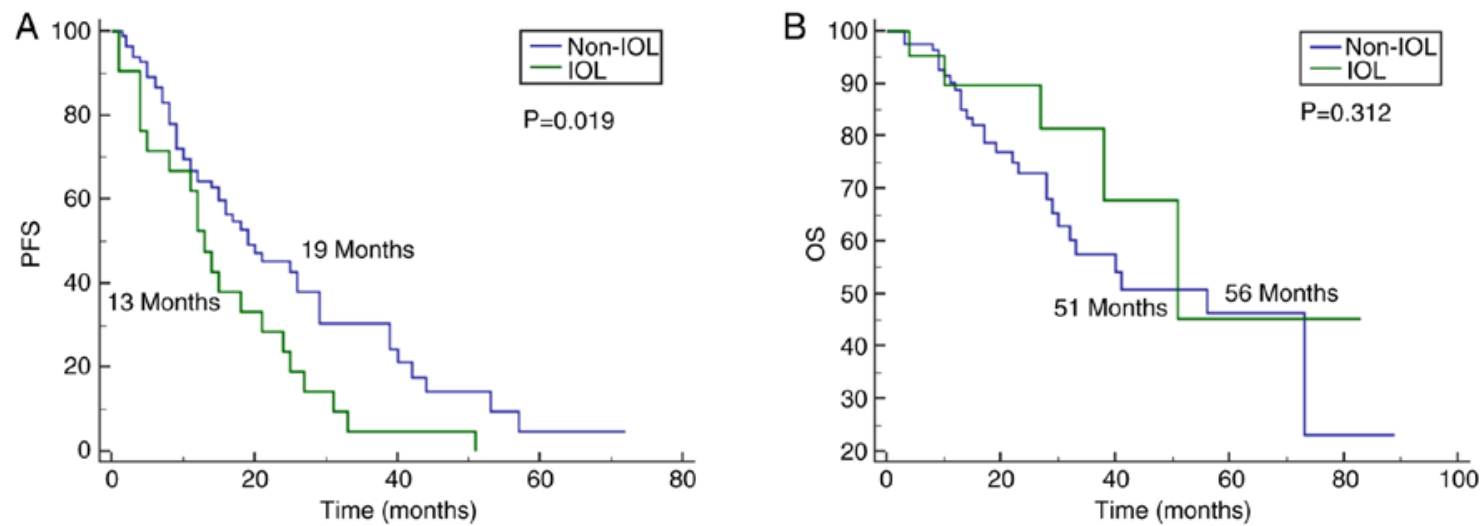

Figure 2. Kaplan-Meier estimates of outcomes by patients with and without intraocular involvement. (A) Progression-free survival. (B) Overall survival. IOL, intraocular lymphoma; non-IOL, no intraocular lymphoma.

was diagnosed with PIOL. The clinical signs of the patients with IOL are summarized in Table II.

Treatment. All the patients were treated with HD-MTX with or without WBRT; in some cases, this treatment was combined with rituximab, IDA and VM-26. A total of 15 patients in the IOL group and 66 patients in the non-IOL group responded to the HD-MTX-based therapy. The overall response (OR) rates were 71.4 and $80.5 \%$, respectively $(\mathrm{P}=0.545)$. In total, 15 patients in the IOL group and 57 patients in the non-IOL 
Table II. Clinical signs of patients with intraocular lymphoma.

\begin{tabular}{lc}
\hline Characteristics & $\begin{array}{c}\text { No. of } \\
\text { patients }\end{array}$ \\
\hline Single eye involvement & 10 \\
Bilateral eye involvement & 11 \\
Symptomatic & 16 \\
Asymptomatic & 5 \\
With parenchyma involvement at diagnosis & 20 \\
Without parenchyma involvement at diagnosis & 1 \\
Intraocular involvement initially & 7 \\
Intraocular involvement during disease progression & 14 \\
\hline
\end{tabular}

Table III. Outcomes of the HD-MTX-based therapy in the IOL and non-IOL patients.

\begin{tabular}{lccc}
\hline & Non-IOL (\%) & IOL (\%) & P-value \\
\hline OR rate, \% & 80.5 & 71.4 & 0.545 \\
CR rates, \% & 69.5 & 71.4 & 0.864
\end{tabular}

HD-MTX, high-dose methotrexate; IOL, intraocular lymphoma; OR, overall response; CR, complete remission.

group achieved complete remission $(\mathrm{P}=0.864)$. No significant differences were observed between the responses of the 2 groups (Table III).

A total of 16 patients received intravitreal injections of MTX at a dose of $400 \mu \mathrm{g} / 0.1 \mathrm{ml}$ after presenting with intraocular involvement. From this group, 15 experienced a clearance of intraocular tumor cells following $\leq 15$ cycles of intravitreal MTX injections. One patient experienced disease progression in both the eyes and in the calvarium after 7 injection cycles. The main complication of this treatment was reversible corneal injury, which usually occurred after the first 8 weeks of injections. Other complications included complicated cataracts, increased tension, vitreous hemorrhage and optic atrophy.

Disease relapse. Of the 21 patients with IOL, 15 (71.4\%) experienced disease relapse. In total, 3 patients had isolated brain parenchymal relapse, 6 patients had isolated eye relapse, and 6 patients had relapses concurrently involving the brain parenchyma and the eyes. In the non-IOL group, 38 patients (46.3\%) had brain relapse $(\mathrm{P}=0.04)$ (Table I). The 2-year PFS rates of the IOL and non-IOL groups were 23.8 and $23.2 \%$, respectively $(\mathrm{P}=0.951)$. The 2 -year OS rates of the IOL group and non-IOL group were 61.9 and $41.4 \%$, respectively $(\mathrm{P}=0.093)$.

Survival data. The median follow-up periods of the IOL and non-IOL groups were 27 months (ranging from 4 to 83 months) and 20 months (ranging from 3 to 89 months), respectively $(\mathrm{P}=0.842)$. The median PFS in the IOL group was 13 months (95\% CI 9.6-16.3), whereas that in the non-IOL group was 19 months (95\% CI 11.2-26.8; $\mathrm{P}=0.019)$ (Fig. 2A).
The median PFS in the IOL group was significantly shorter compared with the median PFS of patients without intraocular involvement. The median OS of the patients with IOL was 51 months vs. 56 months for the patients without IOL (non-IOL patients; 95\% CI 37.2-74.8; $\mathrm{P}=0.312$ ). No significant difference was found between the 2 groups (Fig. 2B). A univariate analysis identified intraocular involvement as the only factor predictive of PFS (Table IV) and age $(\geq 60)$ as the only factor predictive of OS. Furthermore, a multivariate analysis using backward stepwise selection and including the factors with a P-value $<0.20$ from the univariate analyses, demonstrated that intraocular involvement was a significant risk factor for PFS (Table V) and age ( $\geq 60$ years) was a significant risk factor for OS (Table VI). The risk of death was not affected by intraocular involvement.

\section{Discussion}

In this study, we prospectively evaluated the prognostic value of intraocular involvement in patients with PCNSL. The median follow-up time across both groups was 21 months. The relapse rate of the patients in the IOL group was significantly higher than that of the patients in the non-IOL group. Additionally, a significantly poorer PFS was observed in the IOL group compared with the non-IOL group. Nonetheless, there was no significant difference in the OS rate between the 2 groups.

The survival outcomes of patients with PCNSL with intraocular involvement are controversial. The PFS and OS rates in the reported studies vary widely and have clear variations. The main reason for these different survival outcomes is the heterogeneity of both the inclusion criteria and patient evaluation. Furthermore, the diagnostic criteria for IOL varies across different studies due to a lack of standardization. Grimm et al (14) reported that patients with PCNSL with and without intraocular involvement had similar PFS and OS rates; however, their study only included patients with ocular and parenchymal involvement at diagnosis. Kreher et al (15) demonstrated that patients with IOL had significantly inferior PFS and OS rates compared with non-IOL patients. In this study, not all patients received an ophthalmological evaluation. In the IOL group in this study, we included patients who presented with intraocular involvement either at initial diagnosis or during the course of the disease. Patients with IOL had an inferior PFS, but similar OS compared with those in the non-IOL group, and this difference may have been obtained for the following reasons: i) Many other factors may influence OS; ii) we changed systemic therapeutic regimens after disease relapse, which may improve the outcomes to a certain extent; iii) eye-specific therapy was used in some patients with IOL, which helped decrease the additional tumor burden; and iv) the median follow-up time was too short to identify the differences. Due to the rarity of the disease, further studies are warranted to confirm our results.

This study demonstrated that $20.4 \%$ of patients with PCNSL presented with intraocular involvement at some point during the study, which was within the range reported by other studies $(16,17)$. Although HD-MTX-based therapy improved the prognosis of patients with PCNSL, up to $60 \%$ of patients PCNSL will eventually relapse (18). Late relapses account for $4 \%$ of all recurrences in the PCNSL population (19). More 
Table IV. Univariate analysis for risk factors of PFS and OS.

\begin{tabular}{|c|c|c|c|c|c|c|}
\hline \multirow[b]{2}{*}{ Characteristic } & \multicolumn{3}{|c|}{ PFS } & \multicolumn{3}{|c|}{ OS } \\
\hline & HR & $95 \%$ CI & P-value & HR & $95 \% \mathrm{CI}$ & P-value \\
\hline Female & 0.992 & $0.610-1.612$ & 0.973 & 1.341 & $0.680-2.643$ & 0.397 \\
\hline Age, $\geq 60$ years & 1.109 & $0.668-1.839$ & 0.689 & 2.004 & $1.009-3.981$ & 0.047 \\
\hline KPS $<40$ & 1.225 & $0.764-1.965$ & 0.399 & 1.148 & $0.577-2.811$ & 0.695 \\
\hline Radiotherapy & 0.872 & $0.548-1.389$ & 0.566 & 1.318 & $0.674-2.576$ & 0.419 \\
\hline Multiple lesions in brain & 1.368 & $0.859-2.177$ & 0.187 & 1.758 & $0.882-3.501$ & 0.109 \\
\hline Biopsy & 0.968 & $0.622-1.576$ & 0.968 & 1.417 & $0.708-2.835$ & 0.325 \\
\hline Elevated LDH & 1.139 & $0.612-1.139$ & 0.612 & 1.083 & $0.529-2.215$ & 0.827 \\
\hline Elevated $\beta 2-\mathrm{MG}$ & 0.954 & $0.574-1.585$ & 0.857 & 1.481 & $0.749-2.928$ & 0.259 \\
\hline Meningeal involvement & 1.756 & $0.908-3.398$ & 0.094 & 1.571 & $0.660-3.738$ & 0.307 \\
\hline Ki-67 $\geq 80$ & 0.944 & $0.487-1.831$ & 0.866 & 0.568 & $0.204-1.585$ & 0.280 \\
\hline Intraocular involvement & 1.804 & $1.083-3.004$ & 0.023 & 0.619 & $0.240-1.599$ & 0.322 \\
\hline
\end{tabular}

PFS, progression-free survival; OS, overall survival; HR, hazard ratio; CI, confidence interval; KPS, Karnofsky performance scores; LDH, lactate dehydrogenase; $\beta 2-\mathrm{MG}, \beta 2$-microglobulin.

Table V. Multivariate analysis for the risk factors of progression-free survival (PFS).

\begin{tabular}{lccc}
\hline & \multicolumn{3}{c}{ PFS } \\
\cline { 2 - 4 } Characteristic & HR & $95 \%$ CI & P-value \\
\hline Intraocular involvement & 2.600 & $1.255-5.376$ & 0.010 \\
Meningeal involvement & 1.842 & $0.914-3.704$ & 0.087 \\
Multiple lesions in brain & 1.543 & $0.746-3.195$ & 0.242 \\
\hline
\end{tabular}

HR, hazard ratio; CI, confidence interval.

Table VI. Multivariate analysis for the risk factors of overall survival (OS).

\begin{tabular}{llll}
\hline & \multicolumn{3}{c}{ OS } \\
\cline { 2 - 4 } Characteristic & HR & $95 \%$ CI & P-value \\
\hline Age, $\geq 60$ years & 2.183 & $1.091-4.386$ & 0.027 \\
Multiple lesions in brain & 1.923 & $0.958-3.861$ & 0.066 \\
\hline
\end{tabular}

HR, hazard ratio; CI, confidence interval.

and more studies are now focused on the prognostic factors of PCNSL. Kim et al (20) demonstrated that age and the Memorial-Sloan Kettering Cancer Center prognostic score were predictive of survival. In the present study, we demonstrated that patients with intraocular involvement had a higher relapse rate. In accordance with the findings of this study, Ferreri et al (21) reported that the prognosis of PIOL with cerebral involvement was poor and that the 2-year OS rate was $39 \%$.
The diagnosis of IOL is challenging and misdiagnoses are not uncommon. Research into this disease requires precise diagnostic approaches and collaborations between ophthalmologists, pathologists and oncologists. Common ophthalmological examinations, such as OCT and flow cytometry, may be sensitive and valuable methods, but may not result in a definitive diagnosis. Cytological studies of vitreous biopsies are the mainstay of diagnoses (22). In the present study, we selected non-invasive ophthalmological examinations for initial detection in suspected patients. Diagnosis was then confirmed by histopathological examinations. This alleviated unnecessary injury and improved the accuracy of the diagnosis. Furthermore, accurate diagnoses require the sufficiently rapid transportation of tissue specimens, good quality specimens, and experienced interpretation (23). In general, tumor cells in the specimens are limited in number and are dispersed throughout the sample. In this study, 5 out of the 21 patients with IOL were asymptomatic when diagnosed, which indicated that ocular symptoms are not a necessity in the diagnosis of IOL. Currently, studies have indicated that a biochemical test of the interleukin (IL)-10/IL-6 cytokine ratio in the vitreous body helps improve the diagnostic efficacy $(24,25)$. We also found that this ratio was higher in the patients IOL than the non-IOL patients (data not shown), which indicated the promise of this ratio for diagnosis.

It is noteworthy that intraocular involvement developed as a recurrent disease during the interval of systemic MTX therapy in 14 patients with IOL. These observations support the notion that chemotherapeutic agents, including systemic MTX, may not adequately penetrate the blood-ocular barrier. The early recognition of risk factors for intraocular involvement may facilitate early detection and prompt treatment. Cho and Yu (26) demonstrated that patients with PCNSL with a higher Ki-67 level were prone to intraocular dissemination. Further studies are required to enhance the early detection of intraocular involvement and to determine which patients will 
require eye-dedicated prophylaxis. The findings of this study indicate that the follow-up ocular examinations during the course of MTX therapy are also essential.

Due to the blood-ocular barrier, systemic HD-MTX is not sufficient to achieve targeted therapeutic concentrations in the eyes. Eye-dedicated therapy, including intravitreal MTX injection and ocular radiation, is a necessity for patients with IOL to locally control their disease. Additionally, Akiyama et al (27) demonstrated that intravitreal MTX combined with systemic high-dose MTX is effective at preventing CNS involvement in PIOL. Although repeated intravitreal MTX injections had many local complications, this therapy often had a satisfactory effect, and the side-effects were well tolerated. Only 1 in 16 patients had disease progression from the left vitreum to the right. Clinical manifestations, such as anterior uveitis and the development of new lesions were observed intracalvarium. Similar to many other studies (28 and Refs. therein), the results of this study demonstrated that repeated intravitreal injections of MTX are essential and safe for the treatment of IOL.

Radiotherapy has also been used for IOL treatment and is considered an effective treatment to control primary lesions. Isobe et al (29) demonstrated that 13 of 15 patients with PIOL treated with radiotherapy reached complete remission. However, there is significant ocular toxicity associated with this treatment, including radiation retinopathy and cataract formation. As a result, radiotherapy is only indicated as a treatment for recurrent IOL after intravitreal MTX injections or in cases where local chemotherapy cannot be used (30). However, Berenbom et al (31) state that intravitreal chemotherapy does not possess a clear advantage and that radiotherapy may still be the most appropriate first-line treatment in most cases.

The present study has several limitations. The small sample size is the major limitation of our study due to the low morbidity rate. As a result, we did not compare the clinical features of patients with IOL at diagnosis and relapse. Second, chorioretinal biopsies should be performed if vitreous samples fail to provide diagnostic tissue. This type of examination increases reliability in diagnosing and excluding a PIOL that involves the retina or choroid. However, due to permanent defects in biopsied nerves and irreversible visual impairment, we did not perform this examination.

In conclusion, the present study demonstrates IOL patients have a high risk of disease relapse and a poor PFS, but a similar OS to the non-IOL group. Our results suggest the importance of intraocular evaluation for PCNSL patients. Prospective multicenter studies and collaborative efforts are required to better understand the pathogenesis and optimal treatment for IOL.

\section{Acknowledgements}

Not applicable.

\section{Funding}

This study was supported by grants from the China National Clinical Key Subject Construction Foundation (Oncology Department, 2013), Shanghai Hospital Development Center (grant no. 16CR2043B), Natural Science Foundation of China (grant no. 81700123), Beijing Medical and Health foundation (YWJKJJHKYJJ-B17513) and Major Program of Science Foundation of Shanghai Municipal Commission of Health (no. 201540384).

\section{Availability of data and materials}

All data generated or analyzed during this study are included in this published article or are available upon reasonable request from the corresponding authors.

\section{Authors' contributions}

LZ and JL analyzed the patient data and wrote the manuscript. $\mathrm{KC}$ made contributions to the conception and design of the study. TD, YY, YM, HK, ZL, NF, JM and QZ participated in the implementation of the treatment. XX, QW and BC substantially contributed to the study conception and design, reviewed the manuscript and gave final approval of the version to be published. All authors have read and approved the final manuscript.

\section{Ethics approval and consent to participate}

This study received approval from the Ethics Committee of the Institutional Review Board of Huashan Hospital, Fudan University. All the patients voluntarily participated in this study and provided informed consent.

\section{Patient consent for publication}

Not applicable.

\section{Competing interests}

The authors declare that they have no competing interests.

\section{References}

1. Abrey LE, Batchelor TT, Ferreri AJ, Gospodarowicz M, Pulczynski EJ, Zucca E, Smith JR, Korfel A, Soussain C, DeAngelis LM, et al: Report of an international workshop to standardize baseline evaluation and response criteria for primary CNS lymphoma. J Clin Oncol 23: 5034-5043, 2005.

2. Chan CC, Rubenstein JL, Coupland SE, Davis JL, Harbour JW, Johnston PB, Cassoux N, Touitou V, Smith JR, Batchelor TT, et al: Primary vitreoretinal lymphoma: A report from an international primary central nervous system lymphoma collaborative group symposium. Oncologist 16: 1589-1599, 2011.

3. Peterson K, Gordon KB, Heinemann MH and DeAngelis LM: The clinical spectrum of ocular lymphoma. Cancer-Am Cancer Soc 72: 843-849, 1993.

4. Nussenblatt RB, Chan CC, Wilson WH, Hochman J and Gottesman M: International Central Nervous System and Ocular Lymphoma Workshop: Recommendations for the future. Ocul Immunol Inflamm 14: 139-144, 2006.

5. Sung CO, Kim SC, Karnan S, Karube K, Shin HJ, Nam DH, Suh YL, Kim SH, Kim JY, Kim SJ, et al: Genomic profiling combined with gene expression profiling in primary central nervous system lymphoma. Blood 117: 1291-1300, 2011.

6. Kim MM, Dabaja BS, Medeiros J, Kim S, Allen P, Chevez-Barrios P, Gombos DS and Fowler N: Survival outcomes of primary intraocular lymphoma: A single-institution experience. Am J Clin Oncol 39: 109-113, 2016.

7. Wang L, Sato-Otsubo A, Sugita S, Takase H, Mochizuki M, Usui Y, Goto H, Koyama T, Akiyama H, Miura O, et al: High-resolution genomic copy number profiling of primary intraocular lymphoma by single nucleotide polymorphism microarrays. Cancer Sci 105: 592-599, 2014. 
8. Mulay K, Narula R and Honavar SG: Primary vitreoretinal lymphoma. Indian J Ophthalmol 63: 180-186, 2015.

9. Witmer MT: Primary vitreoretinal lymphoma: Management of isolated ocular disease. Cancer Control 23: 110-116, 2016.

10. Stubiger N, Kakkassery V, Gundlach E, Winterhalter S and Pleyer U: Diagnostics and treatment of primary vitreoretinal lymphoma. Ophthalmologe 112: 223-230, 2015 (In German).

11. Rajagopal R and Harbour JW: Diagnostic testing and treatment choices in primary vitreoretinal lymphoma. Retina 31: 435-440, 2011.

12. Cho BJ, Kim DY, Park UC, Lee JY, Yoon YH and Yu HG: Clinical features and treatment outcomes of vitreoretinal lymphoma according to its association with CNS lymphoma. Ocul Immunol Inflamm 26: 365-371, 2018.

13. Ahle G, Touitou V, Cassoux N, Bouyon M, Humbrecht C, Oesterlé H, Baraniskin A, Soussain C, Nguyen-Them L, Gaultier C, et al: Optic nerve infiltration in primary central nervous system lymphoma. Jama Neurol 74: 1368-1373, 2017.

14. Grimm SA, McCannel CA, Omuro AM, Ferreri AJ, Blay JY, Neuwelt EA, Siegal T, Batchelor T, Jahnke K, Shenkier TN, et al: Primary CNS lymphoma with intraocular involvement: International PCNSL Collaborative Group Report. Neurology 71: 1355-1360, 2008.

15. Kreher S, Strehlow F, Martus P, Roth P, Hertenstein B, Röth A, Birnbaum T, Griesinger F, Rauch M, Kanz L, et al: Prognostic impact of intraocular involvement in primary CNS lymphoma: Experience from the G-PCNSL-SG1 trial. Ann Hematol 94 409-414, 2015

16. Jahnke K, Korfel A, Komm J, Bechrakis NE, Stein H, Thiel E and Coupland SE: Intraocular lymphoma 2000-2005: Results of a retrospective multicentre trial. Graefes Arch Clin Exp Ophthalmol 244: 663-669, 2006.

17. Kaburaki T, Taoka K, Matsuda J, Yamashita H, Matsuda I, Tsuji H, Tanaka R, Nakazaki K, Nakamura F, Kamiya K, et al: Combined intravitreal methotrexate and immunochemotherapy followed by reduced-dose whole-brain radiotherapy for newly diagnosed B-cell primary intraocular lymphoma. Br J Haematol 179: 246-255, 2017.

18. Langner-Lemercier S, Houillier C, Soussain C, Ghesquières H, Chinot O, Taillandier L, Soubeyran P, Lamy T, Morschhauser F, Benouaich-Amiel A, et al: Primary CNS lymphoma at first relapse/progression: Characteristics, management, and outcome of 256 patients from the French LOC network. Neuro Oncol 18 : 1297-1303, 2016.

19. Nayak L, Hedvat C, Rosenblum MK, Abrey LE and DeAngelis LM: Late relapse in primary central nervous system lymphoma: Clonal persistence. Neuro Oncol 13: 525-529, 2011.
20. Kim JE, Yoon DH, Kim S, Lee DH, Kim JH, Yoon YH, Chi HS, Lee SW, Park CS, Huh J and Suh C: Relapse pattern and prognostic factors for patients with primary central nervous system lymphoma. Korean J Hematol 47: 60-66, 2012.

21. Ferreri AJ, Blay JY, Reni M, Pasini F, Gubkin A, Tirelli U, Calderoni A, Zucca E, Cortelazzo S, Chassagne C, et al: Relevance of intraocular involvement in the management of primary central nervous system lymphomas. Ann Oncol 13: 531-538, 2002.

22. Fend F, Ferreri AJ and Coupland SE: How we diagnose and treat vitreoretinal lymphoma. Br J Haematol 173: 680-692, 2016.

23. Coupland SE, Bechrakis NE, Anastassiou G, Foerster AM, Heiligenhaus A, Pleyer U, Hummel M and Stein H: Evaluation of vitrectomy specimens and chorioretinal biopsies in the diagnosis of primary intraocular lymphoma in patients with Masquerade syndrome. Graefes Arch Clin Exp Ophthalmol 241: 860-870, 2003.

24. Sugita S, Takase H, Sugamoto Y, Arai A, Miura O and Mochizuki M: Diagnosis of intraocular lymphoma by polymerase chain reaction analysis and cytokine profiling of the vitreous fluid. JPN J Ophthalmol 53: 209-214, 2009.

25. Chan CC, Whitcup SM, Solomon D and Nussenblatt RB: Interleukin-10 in the vitreous of patients with primary intraocular lymphoma. Am J Ophthalmol 120: 671-673, 1995.

26. Cho BJ and Yu HG: Risk factors for intraocular involvement in patients with primary central nervous system lymphoma. J Neurooncol 120: 523-529, 2014.

27. Akiyama H, Takase H, Kubo F, Miki T, Yamamoto M, Tomita M, Mochizuki M, Miura O and Arai A: High-dose methotrexate following intravitreal methotrexate administration in preventing central nervous system involvement of primary intraocular lymphoma. Cancer Sci 107: 1458-1464, 2016.

28. Frenkel S, Hendler K, Siegal T, Shalom E and Pe'Er J: Intravitreal methotrexate for treating vitreoretinal lymphoma: 10 years of experience. Br J Ophthalmol 92: 383-388, 2008.

29. Isobe K, Ejima Y, Tokumaru S, Shikama N, Suzuki G, Takemoto M, Tsuchida E, Nomura M, Shibamoto $Y$ and Hayabuchi N: Treatment of primary intraocular lymphoma with radiation therapy: A multi-institutional survey in Japan. Leuk Lymphoma 47: 1800-1805, 2006.

30. Tropinskaya OF, Vetlova ER, Serova NK, Golanov AV and Fil'Chenkova NA: Radiotherapy of primary intraocular lymphoma associated with primary central nervous system lymphoma. Zh Vopr Neirokhir Im N N Burdenko 80: 74-81, 2016 (In English, Russian).

31. Berenbom A, Davila RM, Lin HS and Harbour JW: Treatment outcomes for primary intraocular lymphoma: Implications for external beam radiotherapy. Eye 21: 1198-1201, 2007. 